\title{
IDENTIFICATION QTL OF LEAVES DIFFUSE REFLECTANCE INDICES IN SPRING HEXAPLOID BREAD WHEAT (Triticum aestivum L.)
}

\author{
Chesnokov Yu. ${ }^{l}$, Kanash Elena ${ }^{1}$, Mirskaya Galina ${ }^{1}$, Kocherina Nataliya ${ }^{l}$, \\ Rusakov D. ${ }^{1}$, Lohwasser Ulrike ${ }^{2}$, Börner Andeas ${ }^{2}$ \\ ${ }^{I}$ Agrophysical Research Institute, St. Petersburg, Russia \\ ${ }^{2}$ Leibniz Institute of Plant Genetics and Crop Plant Research, Gatersleben, Germany \\ e-mail: yuv_chesnokov@agrophys.ru
}

To date, QTL (quantitative trait loci) mapping is one of the main approaches actively used to study interactions between the genotype and the environment. Hereditability, which represents a part of the total phenotypic variability caused by genetic differences, is used in this approach to determine whether a trait can be used for mapping of genes or chromosome loci that determine this trait. QTL mapping of diffuse reflectance indices of laminas in bread wheat (Triticum aestivum L.) has been first performed under controlled conditions of a regulated agroecobiological testing ground in the presence or absence of nitrogen fertilizers. Contact and remote optic technologies were used earlier for the development and implementation of precision agriculture technologies based on the phenotyping of spring wheat; they included a quantitative assessment of plants' need for the main mineral nutrients (nitrogen, phosphorus, and potassium) and water. Results of our earlier studies showed that the conclusion about the suppression of plants and deterioration of their physiological state caused by the presence of a growth-limiting stressor may be made via the measurement of the reduction of a chlorophyll index determined for each crop and cultivar under optimal conditions. Optical indices chosen for the study determine a range of important characteristics, such as the content of chlorophylls and anthocyanins, carotenoid/chlorophyll ratio, photochemical activity of the photosynthetic apparatus, light scattering on a lamina, assimilating leaf surface area, and grain productivity. The reliability of a correlation between the revealed loci and the polymorphism of certain traits was assessed based on a threshold value of the likelihood ratio of the logarithm of the odds or the LOD score. For each experiment, a separate QTL analysis was carried out for each trait, and the trait variation degrees $\left(R^{2}\right)$ explained by the corresponding QTL data were determined. A significance of each LOD was estimated by the permutation test (1000 repeats). Only loci with LOD $\geq 3.0(P<0.001), 2 \leq$ LOD $<3(P<0.01)$, and $1.5 \leq$ LOD $<2(P<0.1)$ were taken into account. In total, 31 QTLs have been mapped. The performed study showed that all six physiological reflectance indices assessed in two parallel experiments demonstrated unstable localization of the corresponding QTLs on the linkage groups. A significant correlation has been revealed between the introduction of a nitrogen fertilizer and the five of six optical characteristics of the photosynthetic apparatus activity in bread wheat. The only exception is the reflectance index for near-infrared radiation $(800 \mathrm{~nm})$, which depends on the structural features of leaf tissues. No statistically significant correlation has been revealed between the thousand-grain weight and spectral characteristics of the diffuse reflectance of the lamina measured at the booting stage. However, a significant correlation between the number of grains formed in the spike of the main stalk and the traits characterizing activity of the photosynthetic apparatus (reflectance indices, leaf area) has been observed. Results of the performed variance, correlation, and QTL analyses confirm each other indicating reliability of the revealed effect of nitrogen nutrition level on the manifestation of the studied reflectance indices in bread wheat under strictly controlled conditions of an agroecobiological testing ground. Application of noninvasive optical methods provides a high-throughput assessment of photosynthetic intensity in plants and, therefore, can be used for efficient selection of promising wheat genotypes with high grain productivity under both controlled and field conditions. They provide a high-throughput analysis of phenotypical traits as well as the study of relationships between the phenotype and genotype and their variability during realization of the evolutionary adaptive and physiologo-genetic "genotypeenvironment" interaction.

The study was supported by the Russian Foundation for Basic Research (16-04-00311a). 